\title{
Nanocomposite Pattern-Mediated Magnetic Interactions for Localized Deposition of Nanomaterials
}

\author{
Despina Fragouli, ${ }^{* \dagger}{ }^{\dagger}$ Bruno Torre, ${ }^{\dagger}$ Francesca Villafiorita-Monteleone, ${ }^{\dagger \dagger}$ Athanasia Kostopoulou, ${ }^{\S}$ \\ Gabriele Nanni, ${ }^{\dagger}$ Andrea Falqui, ${ }^{\perp}$ Alberto Casu, ${ }^{\perp}$ Alexandros Lappas, ${ }^{\circledR}$ Roberto Cingolani, ${ }^{\prime}$ \\ and Athanassia Athanassiou* ${ }^{*}$

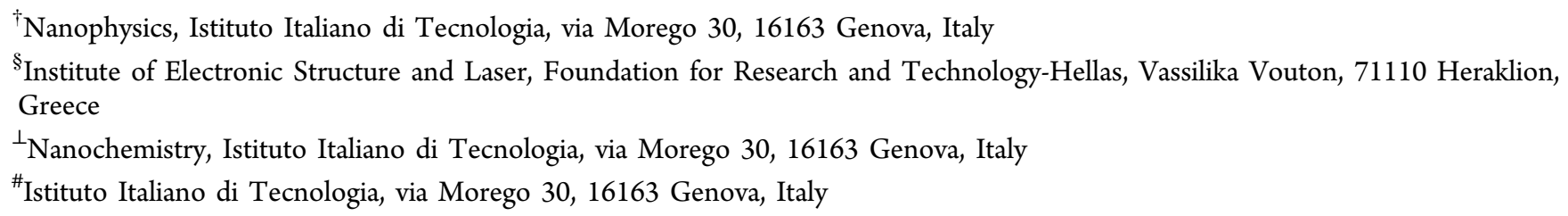

Supporting Information

\begin{abstract}
We present a method to create, align, and locate magnetic wires throughout and on the surface of patterned polymer matrices, following the magnetophoretic transport and self-assembly of ferromagnetic nanoparticles under a static magnetic field during laser photopolymerization of monomer/nanoparticle casted solutions. The resulting films have the ability to attract and immobilize small quantities of magnetic nanomaterials locally on the ferromagnetic wires, as proved by a detailed topography study. Magnetic studies on the films before and after the spontaneous deposition, demonstrate that the deposited nanomaterials alter significantly the magnetic character of the system, making thus possible their macroscopic identification. This offers the possibility to realize sensing devices based on hybrid materials with magnetic properties.
\end{abstract}

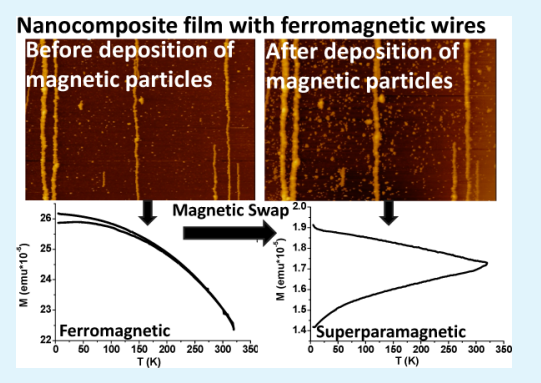

KEYWORDS: magnetophoretic transport, photopolymerization, nanocomposites, patterns, magnetic interactions

\section{INTRODUCTION}

The preparation of organic-based materials possessing superior magnetic properties, comparable with those of fully inorganic systems, is still a challenge. Toward this direction, nanocomposites comprising polymeric matrices containing inorganic magnetic particles, exhibiting modular composition and functionality, have been developed and successfully used as magnetic recording media, ${ }^{1}$ sensors, ${ }^{2,3}$ actuators, ${ }^{4,5}$ MEMS, ${ }^{6}$ electromagnetic wave absorbers, ${ }^{7}$ etc. For many of these applications, the realization of high density and spatially ordered arrays of nanowires into polymeric films ${ }^{8}$ is critical, in order to develop systems with novel collective anisotropically enhanced properties, e.g., mechanical, ${ }^{9}$ electrical, ${ }^{10}$ and magnetic. ${ }^{11}$ In this context, a commonly used strategy is the assembly of nanoparticles (NPs) in polymer or prepolymer solutions in the presence of an external magnetic field (B) applied during the evaporation of the solvent or the curing of the polymer. ${ }^{12,13}$ The produced nanocomposites contain homogeneously distributed wirelike structures throughout the polymeric matrix. To expand the geometrical diversity of the formed structures, recent works ${ }^{11,14,15}$ demonstrate the fabrication of magnetic wires, following the magnetophoretic transport and self-assembly of superparamagnetic NPs into a polymer matrix, with their dimensions and periodicity efficiently controlled by the type of the polymeric matrix, the strength of $B,{ }^{11,14}$ and their localization in the bulk of the film defined by the $B$ application time ${ }^{11}$ or intensity ${ }^{15}$ during the polymer curing. In such ways, specific structural limitations were overpassed. Nevertheless, the localization of such nanocomposite films and the selectivity in their shape and size on specifically defined areas of a solid substrate remained a challenge. If such constraint is overpassed, the successful fabrication of magnetic nanocomposite patterns with collective magnetic properties can pave the way to their incorporation in various magnetic devices.

Herein, we propose a way to go beyond this limit by the realization of magnetic nanocomposites following the magnetophoretic transport and self-assembly of ferromagnetic NPs (FNPs) under a static $B$ during a laser photopolymerization process. The photopolymerization does not affect the FNPs alignment, and most importantly, it permits the creation of patterned nanocomposite films of various shapes that can be selectively defined by the spot of the utilized laser beam. Combined to the FNPs magnetophoretic assembly process, the presented method consent the formation of polymeric patterns that contain wirelike structures, with well-defined collective magnetic properties, exposed on their surface. The fabricated films have the ability to attract magnetic nanomaterials, which

\footnotetext{
Received: April 30, 2013

Accepted: July 9, 2013

Published: July 22, 2013
} 
spontaneously assemble onto the exposed wires, without any external driving field. As a proof of concept, we monitor the spontaneous assembly of superparamagnetic nanoclusters (SCNCs), by the direct microscopic observation of the ferromagnetic wires, and above all by monitoring the notable change in the magnetic behavior of the system before and after the SCNCs deposition. In fact, the magnetic study of the samples reveals that the assembled SCNCs, shield magnetically the underlying ferromagnetic wires inducing a significant alteration in the final magnetic properties of the prepared nanocomposites. Therefore, the presented procedure offers the possibility to create, align, and locate magnetic wires throughout and at the surface of patterned polymer matrices, and eventually to exploit the magnetic properties of the resulting materials to easily immobilize very small structures with magnetic behavior, such as proteins and NPs. This is a promising route toward the realization of sensing devices based on hybrid materials with magnetic properties.

\section{RESULTS AND DISCUSSION}

The photopolymerized nanocomposite coatings were formed after the irradiation with pulsed UV laser light $\left(\lambda_{\mathrm{ex}}=355 \mathrm{~nm}\right.$, energy density $\left.=10.5 \mathrm{~mJ} \mathrm{~cm}^{-2}\right)$ of casted chloroform solutions of methylmethacrylate (MMA) monomer, a photoinitiator (PI) and iron carbide FNPs under a weak $B(\sim 160 \mathrm{mT})$. Such process results in the magnetophoretic transport and assembly of the FNPs parallel to the field's direction resulting in the formation of elongated periodic structures, hundreds of micrometers long and few micrometers thick, during the photopolymerization, which causes their immobilization into the formed poly(methyl methacrylate) (PMMA) polymer (Figure 1). In the nonirradiated areas the gel-like monomer

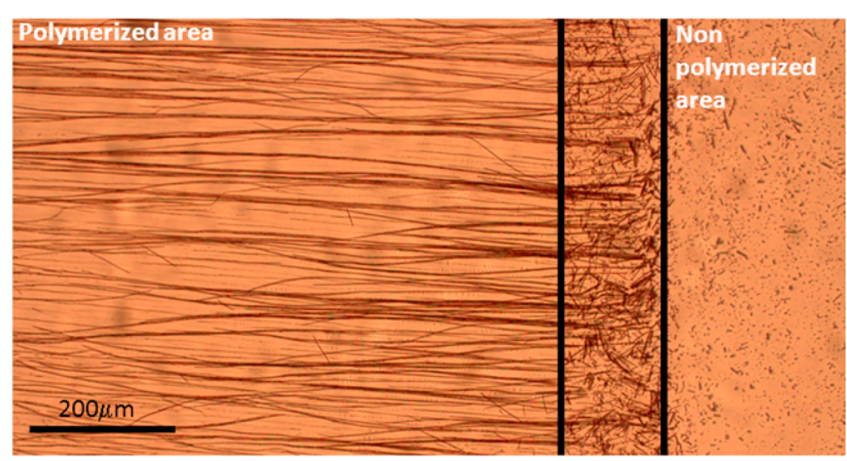

Figure 1. Optical microscope image of the fabricated pattern after the photopolymerization of MMA-PI-FeC film under external $B$. The edge of the laser irradiated part is evidenced between the vertical black lines.

matrix facilitates the disorientation of the structures after the removal of $B$, due to the Brownian motion of the FNPs. In the absence of external $B$ during photopolymerization, the resulting coatings contain FNP aggregates of dimensions in the submicrometer range, without a defined shape and randomly distributed throughout their entire volume (see also Supporting Information, Figure S1)

In Figure 2, atomic force and magnetic force microscopy data of photopolymerized PMMA/FeC samples, acquired using a silicon cantilever coated with a $\mathrm{CrCo}$ magnetic layer, are compared in order to show the structural and functional texture of the produced nanocomposite surfaces. In particular, the topography image of Figure $2 \mathrm{a}$ shows an elongated stripe

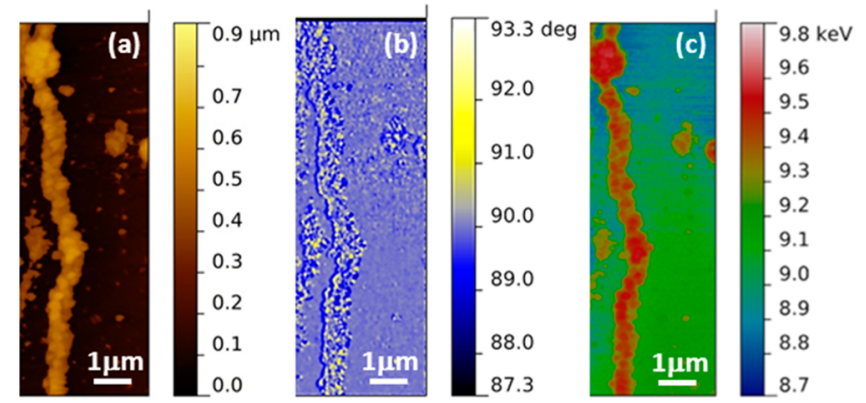

Figure 2. (a) Surface topography, (b) phase lag, and (c) second pass energy loss of the photopolymerized PMMA/FeC nanocomposite.

protruding from a very flat surface, as proved by the surface roughness study (RMS: $140 \mathrm{~nm}$ and $2 \mathrm{~nm}$, respectively, corresponding to the aligned stripes and the polymer region shown also in Figure 1). Such remarkably higher roughness in the stripes' region and the phase image acquired during the first pass (i.e., sensitive to short-range forces) (Figure $2 \mathrm{~b}$ ) indicate that they are protruding and exposed on the films' surface (see the Supporting Information, Figures S2 and S3), as opposed to the nanocomposite films produced by the evaporation process presented in previous studies. ${ }^{11,16}$ Indeed, in the latter case, during the drop casting and solvent evaporation process, the top layer of the film dries very fast resulting in the formation of a very thin nanocomposite layer covering the wires. ${ }^{16}$

This particular bimodal surface distribution increases the effective magnetic surface exposed, with respect to the flat, magnetically inactive one, and is a unique feature of the photopolymerization process. The shape of the magnetic field across the sample's surface is shown by the magnetic signal of energy loss acquired in second pass mode (NAP mode), ${ }^{17}$ which is sensitive to long-range forces (Figure 2c). Such signal demonstrates a uniform behavior in the flat polymeric region and a completely different structured behavior in the stripe's region. The magnetic properties of such texture are stable at room temperature (RT) with no external $B$, indicating a local ferromagnetic behavior of the stripes. In this way, using a relatively small amount of FNPs (10 wt \% with respect to the monomer), it is possible to fabricate a plastic device with highly magnetic character in localized areas of the film.

Such magnetic films can be used as substrates for the subsequent selective assembly and detection of magnetic structures. As a proof of concept, a water solution of iron oxide SCNCs was deposited on the previously structured surface, and after drying the topography changes were studied. Specifically, Figure 3a shows the morphology of the structured film before the SCNCs deposition, where each stripe's width is about $500 \mathrm{~nm}$. Figure $3 \mathrm{~b}$ shows under the same magnification the same stripes after SCNCs deposition, where apart from the presence of few and small SCNC aggregates, the polymeric area remains flat. On the other hand, the stripes' width duplicates reaching the $1 \mu \mathrm{m}$, as shown by the line profiles measured on the same areas. Such observation clearly indicates that the SCNCs assembly occurs almost exclusively onto the ferromagnetic stripes.

The SCNCs assembly occurring spontaneously onto the magnetic stripes of the polymeric film has a dramatic effect on the collective magnetic properties of the latter, as shown by the measurements of the samples' magnetic behavior before and after the SCNCs deposition. In particular, Figure 4a, b reports the magnetization versus temperature curves obtained by zero- 

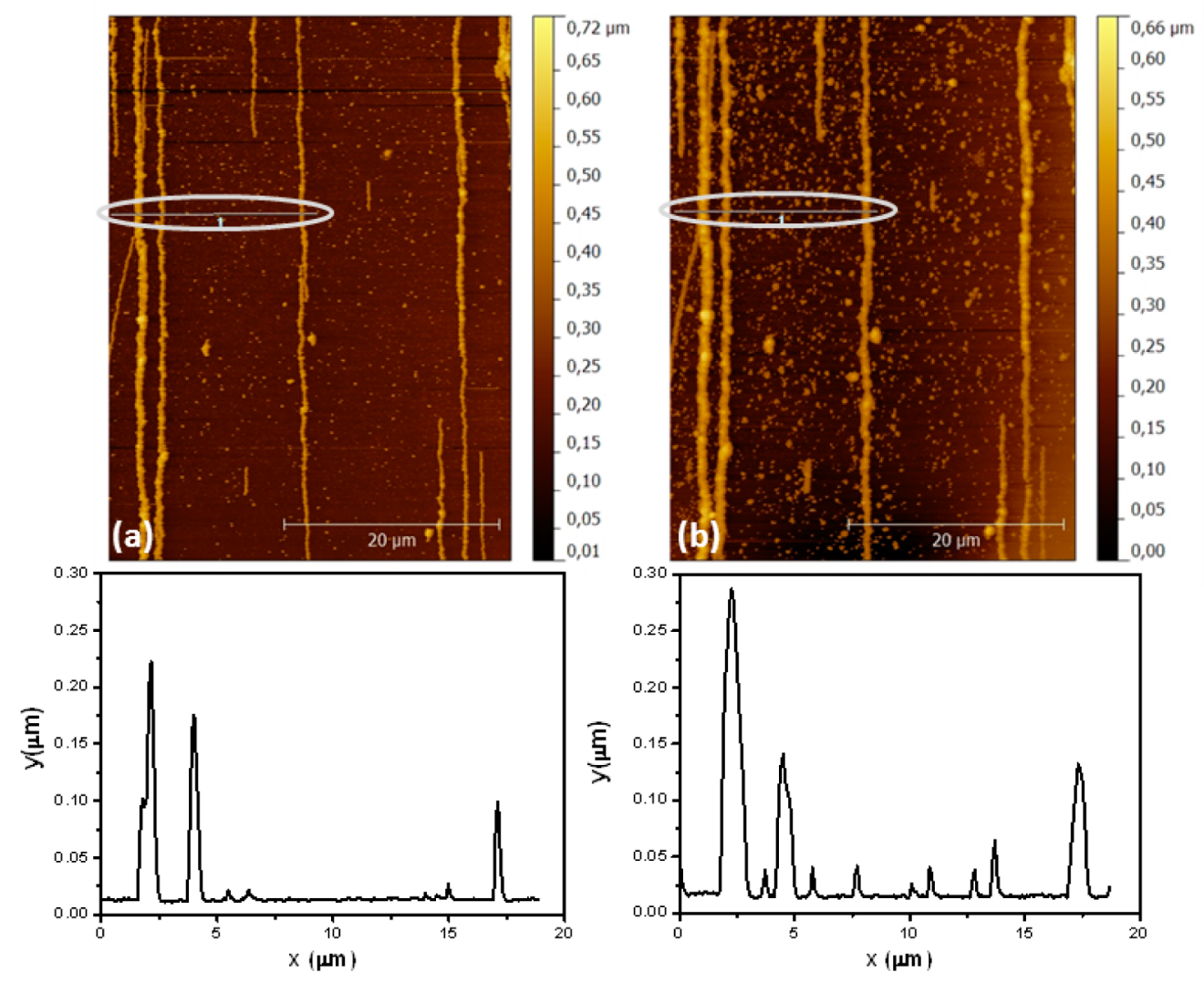

Figure 3. Surface topography of the same area of a structured film (a) before and (b) after the deposition of SCNCs. Insets: Line profiles of the indicated region in both cases.

field-cooled and field-cooled (ZFC-FC) procedure on the same structured film before and after the deposition of SCNCs. The curves clearly indicate that the deposition results in a total screening of the ferromagnetic behavior of the parent samples with stripes (Figure 4a), leading to an overall superparamagnetic behavior (Figure 4b). After their deposition and assembly, the particles giving rise to the magnetic behavior are in the blocked state at RT, as the maximum of the ZFC magnetization has not been reached yet at that temperature. It is worth noting that the decrease in the overall magnetic response of the original ferromagnetic system is about one order of magnitude. The superparamagnetic behavior can be observed also in the ZFC-FC curve measured for the sole SCNCs deposited on a glass substrate (Figure 4c), but in this case the FC curve slightly decreases with the temperature. This fact strongly suggests the occurrence of an antiferromagnetic collective ordering of the SCNCs when cooled in the presence of a low $B(25 \mathrm{Oe})$. This is further supported by the fact that the sole SCNCs are still in the blocked state at RT, as shown in the Supporting Information (Figure S4a). Most importantly, it could explain the strong decrease observed in the magnetic response of the stripes after the SCNCs deposition: when deposited over the stripes, the SCNCs seem to counterbalance the ferromagnetic response of the former, thus partially blocking their magnetic field lines. After the deposition, the overall magnetic response is even lower than that of the SCNCs alone, while the antiferromagnetic ordering observed for the SCNCs disappears. Such observations indicate that the magnetic response is dictated by the small SCNCs fraction constituted by the ones with smaller magnetic size, as well as by the very few ones dispersed on the flat polymeric areas of the film. In fact, these SCNCs can be easily oriented in a low-field regime, resulting in a poor contribution to the blocking of the magnetic field lines of the stripes. To confirm such explanation, a further analysis was performed by calculating the derivative $\mathrm{d}\left(M_{\mathrm{ZFC}}-M_{\mathrm{FC}}\right) / \mathrm{d} T$, which indicates the distribution in the anisotropy energy barrier for each sample and is correlated to the particles magnetic size distribution. ${ }^{18-20}$ The presence of peaks gives an indication on the mean size of the magnetically active SCNCs in each curve (insets, Figure 4). Although the absence of peaks in the top curve (structured sample) confirms a bulklike ferromagnetic behavior of the magnetic stripes, the strong variation in the maxima observed in the other two cases indicates a higher effective magnetic mean size for the sole SCNCs (broad peak at around $180 \mathrm{~K}$ ) with respect to that of the stripes/SCNCs system (sharp peak at $10 \mathrm{~K}$ ). Actually, because the same SCNCs were used in both cases, the change in the effective magnetic mean size should be attributed to the fact that larger SCNCs block the magnetic field lines of the magnetic stripes. Therefore, they do not contribute to the superparamagnetic behavior of the stripes/SCNCs system which is solely attributed to the smaller SCNCs in the low magnetic fields ( $25 \mathrm{Oe}$ ) region. The magnetic response of the stripes/SCNCs system varies greatly in presence of high magnetic fields, which override the "magnetic blocking" operated by the SCNCs on the magnetic stripes. Thus the overall magnetic behavior is mainly dominated by the stronger magnetic character of the ferromagnetic stripes, as shown by the hysteresis loops of the samples registered at RT (see the Supporting Information, Figure S4b and Table S1). 


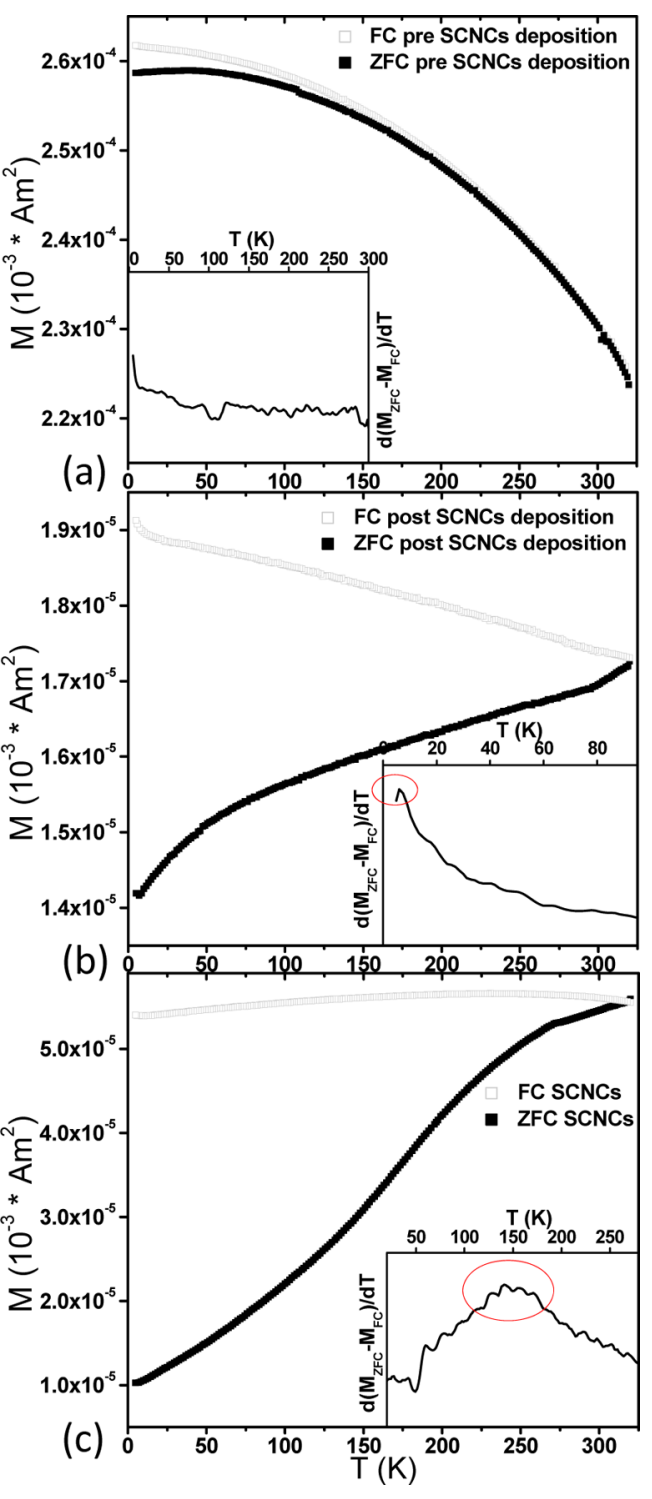

Figure 4. ZFC-FC M(T) curves measured in a magnetic field of 25 Oe for (a) the patterned sample, (b) the patterned sample after SCNCs deposition, (c) the SCNCs on glass substrate. Insets: Calculated anisotropy barrier variation with the temperature for all samples. Red cycles indicate the $T_{B}$ of the mean-sized particles for each case.

\section{CONCLUSIONS}

The above-mentioned study clearly demonstrates the utility of magnetic nanocomposites produced with the proposed method for the fabrication of magnetic sensors. The use of the photopolymerization method for the development of the proposed magnetic composites facilitates their incorporation in devices of different sizes. In addition, the concentration of the FNPs in the form of stripes exposed onto the film surface, because of the magnetophoretic assembly, leads to the distinctive magnetic character of the nanocomposite film. Above all, the fabricated ferromagnetic system has the ability to attract magnetic dust or other magnetic structures without any external driving field. The spontaneously assembled particles can be detected either by direct microscopic observation or, most importantly, by monitoring the remarkable change in the magnetic behavior of the system, which crosses over from ferromagnetic to superparamagnetic. Thus, the presented method paves the way to the fabrication of novel magnetic devices that can easily entrap and detect magnetic substances, in order to be used in various fields ranging from biological to environmental applications. ${ }^{21,22}$

\section{EXPERIMENTAL METHODS}

Materials. All chemicals were used as received. MMA (99\% purity) was purchased from Sigma-Aldrich. The photoinitiator IRGACURE1700 (25\% bis(2,6-dimethoxy benzoyl) 2,4,4-trimethyl-pentylphosphineoxide, $75 \%$ 2-hydroxy-2-methyl-1-phenyl-propan-1-one (DAROCURE1173)) was purchased from Ciba SpecialChem. The ferromagnetic iron carbide (FeC) NPs (FNPs) were purchased from MKNano, with broad size distribution as demonstrated in the Supporting Information, Figure S5, and a mean diameter of $70 \mathrm{~nm}$. Superparamagnetic $\gamma-\mathrm{Fe}_{2} \mathrm{O}_{3}$ colloidal nanoclusters (SCNCs) with mean diameter of $50 \mathrm{~nm}$ (see the Supporting Information, Figure S6), in water were prepared as described elsewhere. ${ }^{23}$ SCNCs are composed by $\gamma-\mathrm{Fe}_{2} \mathrm{O}_{3}$ NPs of mean diameter of $12.7 \pm 1.0 \mathrm{~nm}$ as proved by the XRD analysis presented in the Supporting Information, Figure S7. Furthermore, the chemical nature of the SCNCs has been identified by means of Mössbauer spectroscopy. ${ }^{24}$ Glass microscope slides were used as substrates. All solvents used were purchased from Sigma Aldrich.

Nanocomposites Preparation by Photopolymerization. An homogeneous solution of $89.5 \mathrm{wt} \%$ of MMA, $10 \mathrm{wt} \%$ of FeC NPs and $0.5 \mathrm{wt} \%$ of photoinitiator was prepared in chloroform. $1 \times 1 \mathrm{~cm}^{2}$ glass slides were either put or not in a static $B(\sim 160 \mathrm{mT})$ with a direction parallel to the substrate, generated by two permanent magnets placed at a fixed distance between them. $40 \mu \mathrm{L}$ of the solution was drop casted on each substrate, and the as-prepared samples were irradiated with the third harmonic of a pulsed Nd:YAG laser (QuantaRay GCR 190, Spectra Physics) with an energy density of $10.5 \mathrm{~mJ}$ $\mathrm{cm}^{-2}\left(\lambda_{\mathrm{ex}}=355 \mathrm{~nm}\right.$, pulse duration $=4-6 \mathrm{~ns}$, repetition rate $\left.=10 \mathrm{~Hz}\right)$ for $90 \mathrm{~min}$ at saturated chloroform atmosphere. After the photopolymerization, each sample was washed three times with methanol to remove unreacted monomer and photoinitiator, and then dried under ambient dark conditions.

Deposition of Superparamagnetic Material. The initial concentration of the SCNCs was $4.1 \mathrm{mg} \mathrm{mL}^{-1}$ in water. When this solution was casted on the fabricated films, not any significant preferential deposition onto the magnetic wires was observed (see the Supporting Information, Figure S8). To define the optimum concentration of the SCNCs solution for successful deposition, various trials were conducted. After a systematic study, we found a concentration of $0.7 \mathrm{mg} \mathrm{mL}^{-1}$ to be appropriate for the localized deposition presented herein. $5 \mu \mathrm{L}$ of the SCNCs water solution was drop casted onto the fabricated samples and left to evaporate overnight under ambient conditions. After complete water evaporation, the samples were rinsed with deionized water, in order to remove SCNCs in excess.

Characterization of Samples. Atomic force microscopy and magnetic force microscopy measurements (AFM and MFM) were performed using commercial rectangular silicon cantilever coated with a CrCo layer of $\sim 15 \mathrm{~nm}$ thickness (Asylum Research MFM low moment; magnetization $3 \times 10^{-14} \mathrm{emu}$; coercitivity <400 Oe). The nominal spring constant, $k$, and resonance frequency, $\nu_{0}$, were $2.5 \mathrm{~N}$ $\mathrm{m}^{-1}$ and $75 \mathrm{kHz}$, respectively. Mechanical parameters of free resonance frequency, free oscillation amplitude, quality factor $(Q)$ and $k$ for each cantilever were carefully calibrated on a rigid (nonmagnetic) substrate before each measurement, to obtain energy loss and force gradient signals as previously described. ${ }^{17}$ The samples' morphology and magnetic data are acquired by means of MFP-3D AFM (Asylum Research, Santa Barbara CA) using MFM technique in order to resolve magnetic structure with nanometer resolution. Topography is acquired in AM-AFM mode, whereas magnetic data was acquired in second pass mode using high oscillation amplitude regime. For the images presented the following true imaging parameters are used: free amplitude $A_{0}=280 \mathrm{~nm} ; k=1.68 \mathrm{~N} \mathrm{~m}^{-1}$; $\nu_{0}=68.03 \mathrm{kHz} ; Q=171.1$ and second pass height $=100 \mathrm{~nm}$, corresponding to a minimum elevation of $20 \mathrm{~nm}$. Second pass mode is 
performed in amplitude modulation mode for energy loss imaging. Measurements of static magnetizations and hysteretic behavior are performed on a Quantum Design MPMS SQUID magnetometer, equipped with a superconducting magnet capable to produce fields up to $70 \mathrm{kOe}$. Zero-field-cooled (ZFC) magnetizations were measured by cooling samples in zero magnetic field down to $5 \mathrm{~K}$ and increasing the temperature to $325 \mathrm{~K}$ in an applied field of $25 \mathrm{Oe}$, whereas field-cooled (FC) curves were recorded by cooling from 325 to $5 \mathrm{~K}$ the samples while still keeping the field of 25 Oe. The hysteresis loops were recorded at room temperature with an applied field up to $\pm 70 \mathrm{kOe}$. In all measurements the direction of the magnetic field is parallel to the direction of the magnetic wires.

\section{ASSOCIATED CONTENT}

\section{S Supporting Information}

Microscope image of the nanocomposite film formed without application of any magnetic field. AFM topography and line profile of the assembled nanocomposite film. SEM image of the film. Hysteresis loops of the SCNCs deposited on a glass substrate and of the patterned sample before and after SCNCs deposition. Table containing the values of coercive field, remanent magnetization, and magnetization at high field for all samples. SEM image of the FNPs composing a wire. TEM image and size distribution of the SCNCs. XRD analysis of the SCNCs. AFM image of film after deposition of a highly concentrated solution of SCNCs. This material is available free of charge via the Internet at http://pubs.acs.org.

\section{AUTHOR INFORMATION}

\section{Corresponding Author}

*E-mail: despina.fragouli@iit.it (D.F.); athanassia.athanassiou@ iit.it (A.A.).

\section{Present Address}

${ }^{\dagger}$ F.V-M. is currently at Istituto per lo Studio delle Macromolecole, CNR-ISMAC, Via Bassini 15, 20133 Milano, Italy

\section{Author Contributions}

The manuscript was written through contributions of all authors. All authors have given approval to the final version of the manuscript.

\section{Notes}

The authors declare no competing financial interest.

\section{ACKNOWLEDGMENTS}

The authors thank Ms. R. Rufilli and Dr. M. Allione of Istituto Italiano di Tecnologia for the SEM analysis, and for technical assistance in the irradiation experiments respectively, and $\mathrm{Mr}$. K. Brintakis of Institute of Electronic Structure and Laser, Foundation for Research and Technology-Hellas, Heraklion and Department of Physics, Aristotle University of Thessaloniki, Greece, for the fabrication and characterization of nanoparticles. A.L. acknowledges financial support through the Marie-Curie Transfer of Knowledge program NANOTAIL (Grant no. MTKD-CT-2006-042459).

\section{REFERENCES}

(1) Dai, Q.; Berman, D.; Virwani, K.; Frommer, J.; Jubert, P.-O.; Lam, M.; Topuria, T.; Imaino, W.; Nelson, A. Nano Lett. 2010, 10, 3216-3221.

(2) Leigh, S. J.; Purssell, C. P.; Bowen, J.; Hutchins, D. A.; Covington, J. A.; Billson, D. R. Sens. Actuators, A 2011, 168, 66-71.

(3) Kaushik, A.; Solanki, P. R.; Ansari, A. A.; Sumana, G.; Ahmad, S.; Malhotra, B. D. Sens. Actuators, B 2009, 138, 572-580.

(4) Kim, J.; Chung, S.-E.; Choi, S.-E.; Lee, H.; Kim, J.; Kwon, S. Nat, Mater. 2011, 10, 747-752.
(5) Belardi, J.; Schorr, N.; Prucker, O.; Rühe, J. Adv. Funct. Mater. 2011, 21, 3314-3320.

(6) Pirmoradi, F. N.; Jackson, J. K.; Burt, H. M.; Chiao, M. Lab Chip 2011, 11, 3072-3080.

(7) Huo, J.; Wang, L.; Yu, H. J. J. Mater. Sci. 2009, 44, 3917-3927.

(8) Liu, J.-W.; Liang, H.-W.; Yu, S.-H. Chem. Rev. 2012, 112, 47704799.

(9) Erb, R. M.; Libanori, R.; Rothfuchs, N.; Studart, A. R. Science 2012, 335, 199-204.

(10) Kim, I. T.; Tannenbaum, A.; Tannenbaum, R. Carbon 2011, 49, 54-61.

(11) Fragouli, D.; Buonsanti, R.; Bertoni, G.; Sangregorio, C.; Innocenti, C.; Falqui, A.; Gatteschi, D.; Cozzoli, P. D.; Athanassiou, A.; Cingolani, R. ACS Nano 2010, 4, 1873-1878.

(12) Jestin, J.; Cousin, F.; Dubois, I.; Menager, C.; Schweins, R.; Oberdisse, J.; Boue, F. Adv. Mater. 2008, 20, 2533-2540.

(13) Sorrentino, L.; Aurilia, M.; Forte, G.; Iannace, S. J. Appl. Polym. Sci. 2011, 119, 1239-1247.

(14) Lorenzo, D.; Fragouli, D.; Bertoni, G.; Innocenti, C.; Anyfantis, G. C.; Cozzoli, P. D.; Cingolani, R.; Athanassiou, A. J. Appl. Phys. 2012, 112, 083927:1-8.

(15) Ghosh, S.; Puri, I. K. Soft Matter 2013, 9, 2024-2029.

(16) Fragouli, D.; Torre, B.; Bertoni, G.; Buonsanti, R.; Cingolani, R.; Athanassiou, A. Microsc. Res. Tech. 2010, 73, 952-958.

(17) Torre, B.; Bertoni, G.; Fragouli, D.; Falqui, A.; Salerno, M.; Diaspro, A.; Cingolani, R.; Athanassiou, A. Sci. Rep. 2011, 1, 202:1-8.

(18) Denardin, J. C.; Brand, A. L.; Knobe, M.; Panissod, P.; Pakhomov, A. B.; Liu, H.; Zhang, X. X. Phys. Rev. B 2002, 65, 064422:1-8.

(19) Blanco-Gutierrez, V.; Urones-Garrote, E.; Torralvo-Fernandez, M. J.; Saez-Puche, R. Chem. Mater. 2010, 22, 6130-6137.

(20) Blanco-Gutierrez, V.; Saez-Puche, R.; Torralvo-Fernandez, M. J. J. Mater. Chem. 2012, 22, 2992-3003.

(21) Buzea, C.; Pacheco Blandino, I. I.; Robbie, K. Biointerphases 2007, 2, MR17.

(22) Lecoanet, H.; Leveque, F.; Ambrosi, J.-P. Environ. Pollut. 2003, 122, 229-234.

(23) Kostopoulou, A.; Tsiaoussis, I.; Lappas, A. Photonic Nanostruct. 2011, 9, 201-206.

(24) Unpublished results. 